\title{
The Origin of Optical Rotatory Power
}

\author{
By Prof. T. M. Lowry, C.B.E., F.R.S.
}

\section{$\mathrm{P}$} ROF. MAX BORN'S paper on the "Theory of Optical Rotatory Power", which was read at the Royal Society on February 7 and is published in the Proceedings for May 1, 1935, is of first class interest and importance, since it provides an adequate solution of one of the most difficult problems in optics.

More than a century ago, Fresnel suggested that optical rotatory power was eaused by the unequal velocities of transmission of circularly polarised light of opposite signs ; and by a brilliant piece of experimental work he established the real existence of circular double refraction in quartz; but his tentative suggestion that the optical rotation of rock crystal "cannot be reconciled with complete parallelism of the molecular lines", and must therefore depend on a spiral arrangement of these layers, cannot be extended to optically active liquids. It was therefore left to Pasteur to recognise in the phenomenon of dissymmetry (that is, the inability of a figure to be superposed on its image in a mirror) the characteristic feature of right-handedness and left-handedness, which determines the existence of optical rotatory power in crystals and in liquids, and finds its simplest expression either in a spiral or screw-like structure or alternatively in an irregular tetrahedron.

Equations expressing the influence of wave-length on rotatory dispersion were developed in 1900 by Drude; but these were based primarily on an intuitive recognition of the correct mathematical form of the equations; and, although Drude pointed out that "a dissymmetrically isotropic medium would result if all the molecules were irregular tetrahedra of the same kind", he made no attempt to prove that his conception of ions vibrating in helical paths would actually give rise to optical rotation. So recently as 1916, therefore, Gray was justified in complaining that the chemical and physical theories of optical rotatory power "have not been tied together ; it has not been shown why the asymmetric molecule should give rise to these particular equa. tions".

The missing link was supplied in 1915 by Born's theory of coupled vibrators. The form of coupling suggested by Born is of the simplest and most inevitable character, and has been adopted almost universally by subsequent workers in the same field. It is merely an application of the phenomenon of electrical resonance, and implies that, when an electrically charged particle is set in vibration, for example, by an incident light-wave, it sends out a secondary wave, which acts upon neighbouring particles in just the same way as the original lightwave. The difficulty of the problem of optical rotatory power arises from the fact that rotation of the plane of polarised light is a third-order effect which does not appear until four particles have taken part in this process of resonance. No rotation is therefore produced until the original vibration has been handed on from $A$ to $B, C$ and $D$; and even then the effect is a precarious one, which vanishes if the four particles lie in the same plane, or if in any other manner a plane or centre of symmetry is created, for example, by making two of the particles identical. In these circumstances the calculation of optical rotatory power proved to be such a formidable task that it was only attempted twice in a period of twenty years; and even then one of these laborious calculations of the rotatory power of a crystal was vitiated by a numerical error, which has not yet been put right.

The four coupled vibrators of Born's theory obviously correspond closely with the four dissimilar radicals which give rise to optical rotatory power in the simplest optically active molecules, namely, those molecules which contain an asymmetric carbon atom of the type $C R R^{\prime} R^{\prime \prime} R^{\prime \prime \prime}$; but the calculations of the rotatory power of an asymmetric molecule on the basis of Born's theory are so difficult that they have never even been attempted. Two attempts have been made, however, to simplify this extremely complex problem. Thus S. F. Boys, by ignoring the characteristic frequencies of the vibrators, was able to calculate the rotatory powers of four simple alcohols and amines, from the radii and the refractivities of the radicals; and values of these quantities were

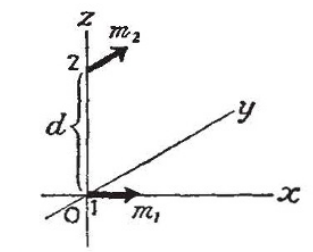

FIG. 1.* Kuhn's simplified model of a dissymmetric molecule.

found which, when inserted in an extremely complex function of the linear dimensions of the molecule, and multiplied by a simple product of the refractivities and other appropriate constants, gave a remarkable concordance between the observed and calculated specific rotations. On the other hand, Werner Kuhn, in 1929, applied Born's theory of coupling to a simplified molecular model consisting of two linear vibrators, moving at right angles to one another (Fig. 1). Optical rotatory power then occurs as a first order effect and can therefore be expressed by much simpler formulæ. He showed that his model gave the formulæ for rotatory dispersion which had already been developed by Drude and Natanson, and that these formulæ then had a self-consistent basis, whereas the spiral vibrators, which Drude suggested as a justification of his formulæ, do not actually give rise to any rotatory power at all.

The postulate of a linear vibrator implies that the ellipsoid of elasticity is reduced to a straight line, so that the particle is able to move to and fro in one given direction, but is so rigidly confined to this linear path that no polarising force, however powerful, is able to make it move in any other direction. Such a conception differs very widely from the relatively flexible electronic systems which are found in chemical

- Figs. 1, 2 and 3 are reproduced by courtesy of Messrs. Longmans, Green and Co., Ltd., from Lowry's "Optical Rotatory Power" (1935) 
molecules. It is perhaps approached rather remotely in substances like benzoquinone, the crystals of which contain giant molecules of indefinite extension in one dimension, along which they exhibit abnormally high refractivities; but substances like amyl alcohol give no hint of any similar strong anisotropy. Moreover, the presence of four dissimilar radicals appears to call inexorably for a system of four vibrators; and calculations based upon a model containing only two vibrators can only have a remote relation to the properties of real molecules of this type. Whilst, therefore, Kuhn's simplified model has provided a new basis for the dispersion formulæ of Drude and Natanson, it does not appear to be capable of direct application to the asymmetric carbon atom, the rotatory power of which must still be treated as a third-order effect in accordance with the original conceptions of Born.

More favourable conditions occur in certain spirocompounds, which are not asymmetric (since they possess a two-fold axis of symmetry) and do not coitain an asymmetric carbon atom, but which can nevertheless be prepared in optically active forms, since they possess the essential property of molecular dissymmetry, that is, the molecule can exist in two mirror image forms which are not superposable on one another. Three such molecules have been studied in this connexion.

I. Erythritoldipyruvic acid, resolved by Boeseken and Felix (Ber., 56, 1855 ; 1928).<smiles>CC1(OC(=O)O)OCC2(CO1)COC(C)(C(=O)O)OC2</smiles>

$[\alpha] D= \pm 4^{\circ}$.

II. Spiroheptanedicarboxylic acid, resolved by Backer and Schurink (Proc. Akad. Wiss., Amsterdam, 31,$370 ; 1928$ ).<smiles>CC(C)(C)CCCCCCC(=O)O</smiles>

III. Diaminospiroheptane resolved by Pope and Jansen.<smiles>CC(C)(C)[13CH3]</smiles>

In each of these compounds the two halves of the molecules are crossed, that is, if the right-hand ring is in the plane of the paper, the left-hand ring is in a plane at right angles to it, since the central carbon atom has a tetrahedral and not a planar configuration. The planes which contain the terminal radicals $\mathrm{COOH}$ and $\mathrm{CH}_{3}, \mathrm{COOH}$ and $\mathrm{H}$, or $\mathrm{NH}_{2}$ and $\mathrm{H}$, are therefore also at right angles to one another. These two rectangular planes can be compared with the directions of the two linear vibrators in Kuhn's original model, and on this basis he has made a detailed study of Boeseken's spiroacid I. For this purpose he has considered the two $-\mathrm{CO} . \mathrm{OH}$ groups as linear vibrators, displaced from the central axis of the molecule in directions at right angles to one another (Fig. 2), and has thus been able to calculate the rotatory power of the molecule.
This figure has the demerit of dealing with only one of the two radicals in the system $\mathrm{CH}_{3}-\mathrm{C}-\mathrm{CO} . \mathrm{OH}$, the 'crossing' of which produces the dissymmetry of the molecule. Born, on the other hand, has deduced a formula for the rotatory power of a system of four vibrators, $A, B, A, B$ (Fig. 3 ), in which both radicals play a part and neither of them need be assumed to be strongly anisotropic. This formula has been applied to the spiro base of Pope and Jansen, where there are good reasons for thinking that spacial dissymmetry, and not the anisotropy of the $\mathrm{H}$ and

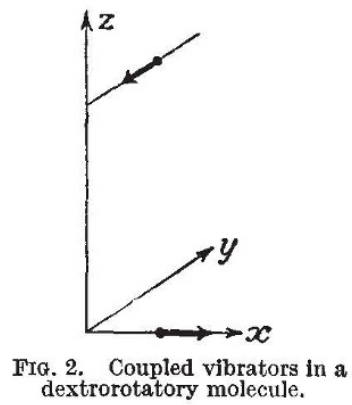

$\mathrm{NH}_{2}$ radicals, is the principal factor in producing the optical rotatory power of the molecule. Results of the right order of magnitude have been obtained; but these are merely guesses, and the principal importance of the formula lies in its application to the relative magnitudes of the rotatory power of related com. pounds, rather than in attempts to predict the absolute rotatory power of any of them. The latter must always be difficult, since the formula involves the eighth power of the distance between the two vibrators, the sixth power of their frequencies and the square of the strengths of their absorption bands. On the other hand, if we compare a series of spirocompounds containing, for example, the radicals

$$
\text { -NH.CO. } \mathrm{NH}_{2}-\mathrm{NH} . \mathrm{CO} . \mathrm{CH}_{3} \text { and }-\mathrm{NH} \text {.CO. } \mathrm{C}_{6} \mathrm{H}_{5}
$$

we can now recognise that their rotatory powers are definite functions of three well-defined properties of

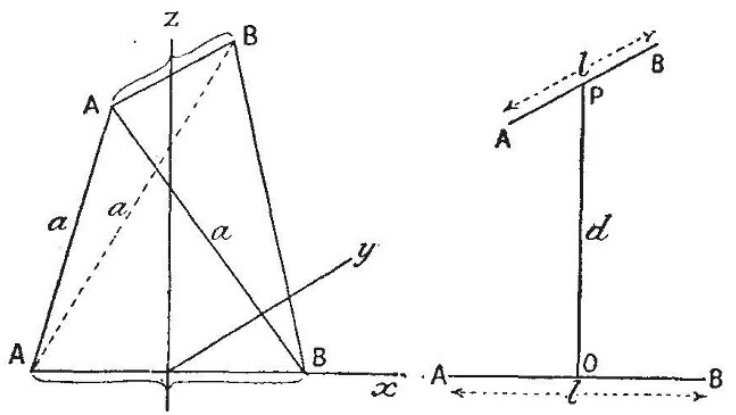

FrG. 3. Born's molecular model.

the radical, and their correlation becomes an experimental problem of very great interest. Unfortunately, the frequencies of the simplest compounds lie in the Schumann region, for which very few data are yet available; but no great increase of complexity is needed in order to bring the frequencies within the range of easier experimental observation; and, on the other hand, the Schumann region is rapidly being opened up to research, so that progress on the lines suggested by Born's theoretical work need not be long delayed. 